\title{
Cross Time-Frequency Analysis of Gastrocnemius Electromyographic Signals in Hypertensive and Nonhypertensive Subjects
}

\author{
Patrick Mitchell, ${ }^{1}$ Debra Krotish, ${ }^{2,3}$ Yong-June Shin, ${ }^{1}$ and Victor Hirth ${ }^{2,3}$ \\ ${ }^{1}$ Department of Electrical Engineering, University of South Carolina, 301 S. Main St. Columbia, SC 29208, USA \\ ${ }^{2}$ Division of Geriatrics, Palmetto Health Richland, 3010 Farrow Road Suite 300A Columbia, SC 29203, USA \\ ${ }^{3}$ School of Medicine, University of South Carolina, 6311 Garners Ferry Rd, Columbia, SC 29209, USA
}

Correspondence should be addressed to Yong-June Shin, shinjune@engr.sc.edu

Received 31 January 2010; Revised 14 May 2010; Accepted 9 August 2010

Academic Editor: Lutfiye Durak

Copyright ( $) 2010$ Patrick Mitchell et al. This is an open access article distributed under the Creative Commons Attribution License, which permits unrestricted use, distribution, and reproduction in any medium, provided the original work is properly cited.

\begin{abstract}
The effects of hypertension are chronic and continuous; it affects gait, balance, and fall risk. Therefore, it is desirable to assess gait health across hypertensive and nonhypertensive subjects in order to prevent or reduce the risk of falls. Analysis of electromyography (EMG) signals can identify age related changes of neuromuscular activation due to various neuropathies and myopathies, but it is difficult to translate these medical changes to clinical diagnosis. To examine and compare geriatrics patients with these gaitaltering diseases, we acquire EMG muscle activation signals, and by use of a timesynchronized mat capable of recording pressure information, we localize the EMG data to the gait cycle, ensuring identical comparison across subjects. Using time-frequency analysis on the EMG signal, in conjunction with several parameters obtained from the time-frequency analyses, we can determine the statistical discrepancy between diseases. We base these parameters on physiological manifestations caused by hypertension, as well as other comorbities that affect the geriatrics community. Using these metrics in a small population, we identify a statistical discrepancy between a control group and subjects with hypertension, neuropathy, diabetes, osteoporosis, arthritis, and several other common diseases which severely affect the geriatrics community.
\end{abstract}

\section{Introduction}

For the older adult population, falls continue to be a threat to morbidity and mortality [1]. Falls accounted for some $\$ 19.5$ billion dollars of health care costs in the United States in 2000 [2], and is one of the significant factors for unintentional injury resulting in death in people aged $65-85$. As the number of older adults increases, the number of fall-related injuries and death is also likely to increase [1]. Over one third of community dwelling adults, aged 65 and older, fall each year $[3,4]$, and the rate of falls increases by $7 \%-17 \%$ for adults 80 years and older [4-6]. Falls are the leading cause of admission to nursing homes and assisted care living facilities [6], and healthcare costs associated with falls are estimated to increase dramatically as fall-related injuries increase for older adults [7-9]. Although the number of injuries and deaths and the economic impact of falls continue to rise, the greatest cost is in the loss of mobility, independence, and autonomy in the later years of life.

Examining methods to identify fall risk factors is paramount to developing a successful strategy to decrease the rate of falls in the United States. A number of studies have examined some of the more common comorbidities that increase fall risk in the geriatric community. Motor impairments rank as one of the most significant factors in falls, resulting from decreases in muscle mass, muscular strength, and muscular power $[10,11]$. Several comorbidities, including neuropathy, diabetes, and high blood pressure, are known to accelerate this degeneration, and thereby increase the risk of falling [12]. In order to quantify this degeneration, we used electromyography, a method long used for assessment of musculoskeletal health and 
diagnosis of specific diseases. To analyze gait associated with detrimental comorbidities objectively, we propose a method using time-frequency analysis of the EMG signals. Since classical EMG analysis uses subjective or intuitive methods to assess gait abnormalities, it is desirable to analyze parameters pertaining to gait in relation to the specific degeneration cause by the aforementioned comorbidities.

Historically, electromyography (EMG), the method of recording the electrical or neurological activity of skeletal muscles, has been particularly useful for determining medical abnormalities and gait deficits, such as neuropathy, Parkinson's, and carpal tunnel syndrome. This paper records gait patterns and EMG signals for comparison across different individuals, with and without the comorbidities in question. To compare subject-to-subject electromyography data accurately, an independent reference of gait is desirable. Therefore, we used a pressure-sensitive walkway, which records time and pressure data that, by an external trigger, is time-synchronized to the recording of the EMG signals, by use of a series of pressure sensors tied together. The EMG signals are recorded using eight surface EMG sensors routed to a wireless transmitter pack, which sends the data to a host computer. This data acquisition process and experimental setup are described in detail in Section 2.1.

However, due to the transient nature of EMG signals [13], we chose an advanced signal processing approach known as time-frequency analysis. Time-frequency analysis techniques have also been applied to EMG signal analysis, in considering the complex phenomenon known as localized muscle fatigue [14], as well as for the assessment and accurate classification of Parkinson's disease [15]. A unique subset of this methodology, known as cross timefrequency analysis, is the primary focus of this paper. This methodology can directly compare two signals and identify discrepancies in the time and frequency domain simultaneously. Previous applications of time-frequency analysis use a thresholding method for signal selection and division, as opposed to our approach, which uses the gait information directly for signal selection. When this method is used in conjunction with cross timefrequency analysis, we can compare EMG data across the same period within the gait cycle. Time-frequency analysis techniques have seen successful application to the biomedical realm, such as in determining the feedback relationships between postural control and visual impairment [16] in the platform test environment. As the knowledge base for application of cross time-frequency analysis grows, the clinical application for this method will become more apparent.

Our experimental setup is introduced in Section 2. The methods of time-frequency analysis and cross timefrequency analysis are discussed in Section 3.1, as well as our unique selection of metrics. Section 4 discusses the results of our initial trial in detail, explaining the application of the metrics to two specific cases, then going on to compare the results of the initial trials. The metrics determined show significant discrepancy on our initial data in a quantitative manner, with differences around eleven percent between hypertensive and nonhypertensive cases.

\section{Electromyography, Gait, and Data Acquisition}

Since muscle activation is not easily apprehended by visual inspection, clinical observation alone does not provide a complete picture of gait coordination. Electromyography can provide information about muscle function within the gait cycle, and the timing of the muscles as they work in selfcoordination [17]. The electrical signals recorded during firing the muscles may be either over-or underactive in the affected muscle groups. We selected four major muscles, including the vastus lateralis, biceps femoris, tibialis anterior, and gastrocnemius due to the predominant role each muscle plays in gait and the muscles' accessibility for data collection.

There is a large range of subjects present in this study, consisting of 57 elderly subjects and 10 younger subjects. Elderly subjects were all over age $65,30 \%$ male, while the younger subjects were all under 35, 90\% male. Recruitment for subjects occurred from a geriatric medical practice and a local retirement community, as well as the graduate department of the publishing school. We obtained approval from the IRB, and all subjects provided informed consent. All participants were cognitively intact, community dwelling healthy older adults, who were ambulatory and living independently. We excluded participants who had a score of less than 25 on the Folstein Mini Mental State Exam (MMSE) [18]. Inclusion in the study was independent of gender, race, or ethnic background. We gathered medical records for each participant from his/her primary care physician. A clinical research nurse reviewed the records and categorized them into two groups according to hypertension diagnosis or no existing comorbidities.

2.1. Data Acquisition. In order to acquire gait-synchronized EMG signals, we used a commercially available wireless surface EMG interface, in conjunction with a pressuresensitive walkway. Figure 1 shows the experimental setup used to collect data. The wireless EMG system uses a lithiumion powered pack (c), which acts as an IEEE $802.11 \mathrm{~b}$ broadcast host. The eight surface EMG sensors (b) route to the pack, where the signals are amplified. The pack samples at $1 \mathrm{kHz}$, with a band-pass filter set from $20 \mathrm{~Hz}-450 \mathrm{~Hz}$, sensor to amplifier length is less than $2 \mathrm{~m}$. When recording, data transfers to a host computer (e) using the Wi-Fi link.

The mat (a) in Figure 1 is a $6 \mathrm{~m}$ long runway with $48 \times 384$ pressure sensors distributed uniformly throughout the mat itself. These sensors route to a central recording unit (f) that samples the sensors at $60 \mathrm{~Hz}$. This sampling records footfall pressure changes in time over the length of the mat. Using a time-synchronization triggering system developed in-house, the signals recorded by the wireless EMG system correspond in time to the information recorded by the walkway.

For the experiment, surface EMGs were applied to the four muscles in each leg by a trained research professional. Figure 1 also shows the location of each of these four sensors, placed on each of the four largest muscle control units responsible for gait, as described in the previous section. The wires were then secured and connected to the wireless 

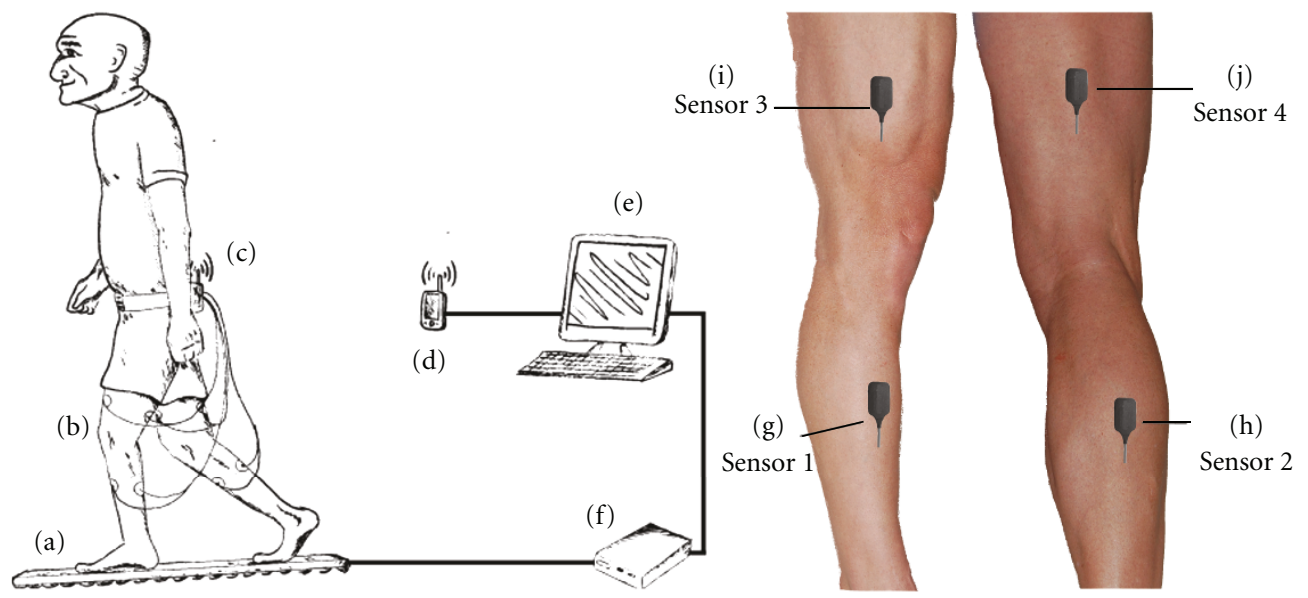

FIGURE 1: Diagram of data collection setup (a) pressure-sensitive walkway, (b) EMG sensors connected to muscles, (c) amplifier and wireless transmitter unit, (d) 802.11 receiver, (e) data-recording computer, (f) walkway data converter and EMG sensor locations, (g) sensor 1: tibialis anterior (h) sensor 2: gastrocnemius (i) sensor 3: vastus lateralis (j) sensor 4: biceps femoris.

transmitter pack. The subjects walked on the pressuresensitive walkway at a normal pace for two trials, then at a faster pace for two additional trials. Subjects began their walk at a designated acceleration line $2 \mathrm{~m}$ before the beginning of the mat and continued to a deceleration line $2 \mathrm{~m}$ after the end of mat.

Figure 2 is a graphical example of a single walk file from a healthy subject. Shown in the first two plots are the left (a) and right (b) gastrocnemius muscle's EMG signals and under these plots is a record of the time-aligned y position information. Due to the four-dimensional (time, $X, Y$, and pressure) nature of the mat, only the time and $Y$-axis (the span of the mat) are displayed (c). The stance phase divisions, marked in red, are determined independent of the EMG information and are located entirely by gait analysis. These divisions align with signal groups for the gastrocnemius contraction, due to its use primarily during the stance phase. The primary wave packets $\left(S_{1}, S_{2}\right)$ correspond to contractions, and their secondary compliments $\left(S_{1}^{\prime}, S_{2}^{\prime}\right)$ correspond to the stance phase of the opposing leg, during which time the gastrocnemius should be in relative disuse.

Once these wave packets are selected in software, we can properly analyze them using time-frequency analysis. By then comparing the wave packets to one another within an individual, we can gather information unique to that subject. Section 3.2 discusses the parameters by which we compare subjects in more detail.

2.2. Use of Gait Synchronization for Signal Division. In order to address the problem of cross-subject muscular activation comparison, we used a footfall synchronization approach. The normal gait cycle employs similar muscular activation regardless of the subject, and is desirable for use as a standard to compare same-muscle activation across subjects. Using the same portion of the gait cycle from subject to subject ensures that upon analysis, the EMG signals are comparable. This method ensures that each cross time-frequency plot is aligned across each subjects' individual gait cycle, and each

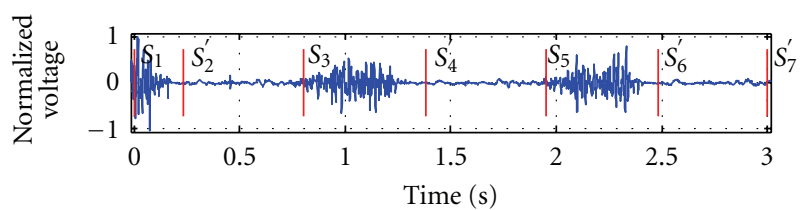

(a)

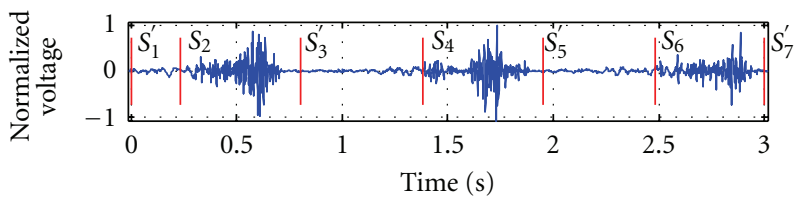

(b)

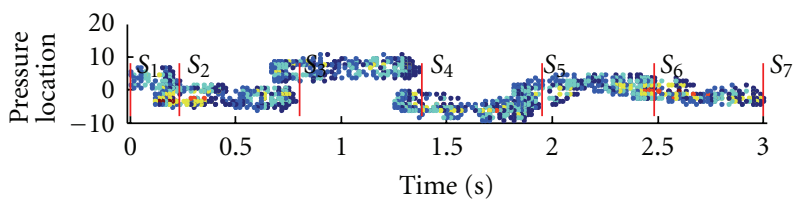

(c)

FIGURE 2: Time-aligned EMG signals of (a) left gastrocnemius (b) right gastrocnemius, and (c) pressure walkway recording, with swing phase divisions shown in red.

signal selected is taken at the same time. After selection, the signals are zero-padded to equalize their lengths to the longer of the two signals, but all signals start immediately at the beginning of each footfall. Figure 2 shows this signal separation in detail within the gastrocnemius muscle, as denoted by the red lines. Each muscle group has different periods of activation, making it desirable to determine the optimal signal selection based on the gait cycle. Figure 3 shows the normalized energy in each of the four muscle groups on one leg through the course of a single healthy gait cycle. Within a single gait cycle, the four major muscle 
groups contract and release to control joint actuation, thus producing locomotion.

The gastrocnemius muscle (c) lends itself to analysis by the nature of its contraction over the course of a single stance phase, as well as its importance to overall gait health. This muscle activates during the push-off stage leading up to the swing phase, actuating the heel and preparing the heel for contact during the next stance phase. During the release of the muscle, the opposing leg's muscle begins activation, causing a very distinct pattern that aligns with footfalls. Thus by lining up wave packet selection with the beginning of each swing phase, we captured the contraction of the muscle over the course of the swing.

\section{Theory of Application of Time-Frequency Analysis to EMG Signals}

3.1. Cross Time-Frequency Analysis. EMG signals have always lent themselves to spectral analysis. Because of their heterogeneous nature, it is desirable to decompose these signals into their primary frequency components. In order to analyze signals in the time and frequency domain simultaneously, we must first divide the signal into smaller segments, and then analyze that segment in the frequency domain. This process is called time-domain windowing, and a timelocalized signal $s_{t}(\tau)$ becomes

$$
s_{t}(\tau)=s(\tau) h(\tau-t)
$$

where $h(t)$ is a window function centered at time $t$. While moving along different time slices, we can determine the frequency content at each slice using the Short-Time Fourier Transform (STFT) [19]. The equation for the STFT is

$$
S_{t}(\omega, t)=\frac{1}{\sqrt{2 \pi}} \int s(\tau) h(\tau-t) e^{-j \omega t} d \tau
$$

By this definition of STFT, one can find the magnitude of the signal at any time and frequency. This representation's resolution on time-frequency domain is highly dependent on proper selection of the window, $h(t)$. Due to the transient nature of electromyography signals as shown in Figure 3, a single window for all cases is not ideal. Therefore, it is desirable to use a representation that is less dependent on proper window selection. The Wigner distribution is one such kernel, but is extremely susceptible to crossterm distortion [19], and when signal characteristics are unknown, this distribution becomes difficult to interpret.

We therefore use the Reduced Interference Distribution (RID), which has the advantages of a time-and frequencydomain representation, while eliminating cross-terms using time-smoothing window as well as a frequency-smoothing window. This distribution has seen previous application in time-frequency analysis when dealing with EMG signals $[13,20]$. These windows reduce the effect that cross-terms have by attenuating the signals where the cross-terms would otherwise occur [21]. The RID is defined as follows:

$$
\operatorname{RID}_{x}(t, \omega)=\int_{-\infty}^{\infty} h(\tau) R_{x}(t, \tau) e^{-j \omega \tau} d \tau,
$$

where $R_{x}(t, \tau)$ is an instantaneous autocorrelation with a time-smoothing window, $h(\tau)$. For our purpose, we chose to use a Hanning frequency-smoothing window. Thus, for a signal $x(t)$ in the time-domain, the instantaneous autocorrelation function is

$$
\begin{aligned}
& R_{x}(t, \tau) \\
& =\int_{-|\tau| / 2}^{|\tau| / 2} \frac{g(v)}{|\tau|}\left(1+\cos \frac{2 \pi v}{\tau}\right) x\left(t+v+\frac{\tau}{2}\right) x^{*}\left(t+v-\frac{\tau}{2}\right) d v,
\end{aligned}
$$

where $g(v)$ is a frequency-smoothing window.

The traditional time-frequency distribution enables us to analyze the time-varying spectral characteristic of a single waveform. However, in this study of gait, it is necessary for us to consider a time-localized cross-correlation between two signals, such as left and right muscle groups responsible for gait. In this paper, the wave packets defined as $S_{i j}$ correspond to the time-domain signal $x(t)$. Shin et al. established a cross time-frequency transformation based on Williams' [21] definition that preserves phase information [22]. To develop this transformation, we begin by using the definition of the instantaneous cross-correlation

$$
R_{x_{1} x_{2}}(t, \tau)=x_{1}\left(\frac{t+\tau}{2}\right) x_{2}^{*}\left(\frac{t-\tau}{2}\right) .
$$

From here we apply the Fourier transform to obtain the cross-Wigner distribution $W_{x_{1} x_{2}}(t, \omega)$,

$$
W_{x_{1} x_{2}}(t, \omega)=\frac{1}{2 \pi} \int R_{x_{1} x_{2}}(t, \tau) e^{-j \omega \tau} d \tau
$$

By taking a 2-D Fourier transform of the desired kernel and then inserting this transformed kernel into the definition of the cross-Wigner distribution, we preserve the phase information contained in the kernel:

$$
J_{x_{1} x_{2}}(t, \omega ; \Phi)=\frac{1}{4 \pi^{2}} \iint W_{x_{1} x_{2}}(u, \xi) \Phi(t-u, \omega-\xi) d u d \xi
$$

where $\Phi(t, \omega)$ is the 2-D Fourier transform of the desired kernel $\phi(\theta, \tau)$ :

$$
\Phi(t, \omega)=\iint \phi(\theta, \tau) e^{-j(\theta t+\tau \omega)} d \theta d t
$$

We finally define the cross time-frequency distribution as follows [22]:

$$
\begin{aligned}
& J_{x_{1} x_{2}}(t, \omega ; \phi) \\
& =\frac{1}{4 \pi^{2}} \iiint x_{1}\left(t-\frac{\tau}{2}\right) x_{2}^{*}\left(t+\frac{\tau}{2}\right) \phi(\theta, \tau) e^{-j \theta t-j \tau \omega+j \tau v} d \theta d \tau d v .
\end{aligned}
$$

By preserving the phase, we can ascertain valuable information that would otherwise be lost, as well as information about the correlation between two signals $x_{1}(t)$ and $x_{2}(t)$. In contrast to the cross-correlation, the cross time-frequency distribution allows us to determine the localized time-and 


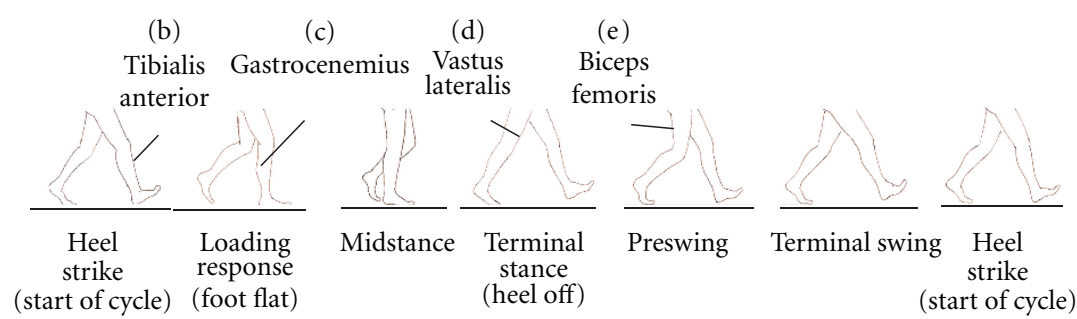

\begin{tabular}{|c|l|l|l|l} 
Stance phase & Swing phase \\
\hline Single gait cycle &
\end{tabular}

(a)

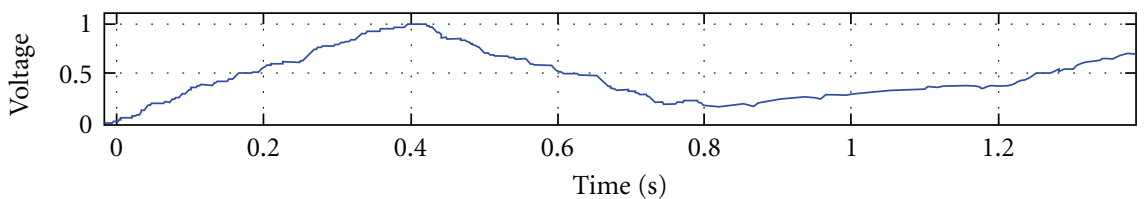

(b)

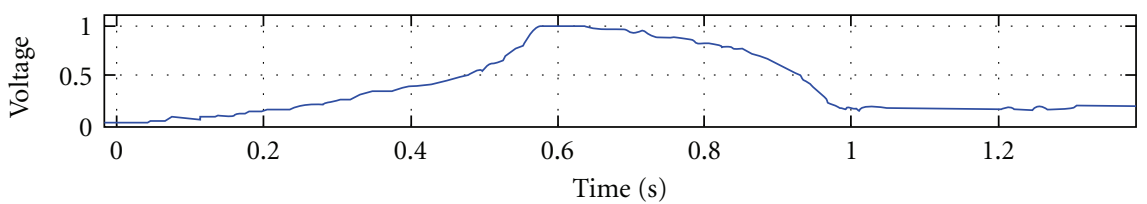

(c)

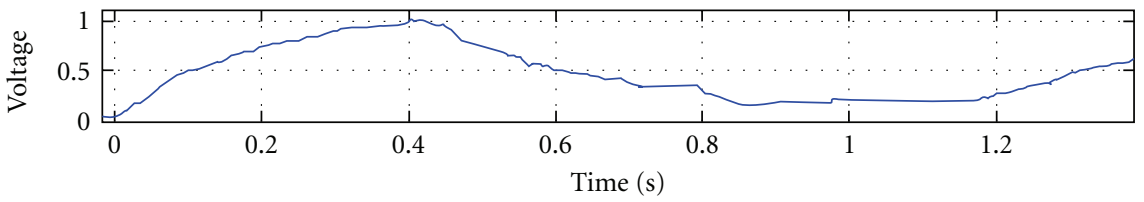

(d)

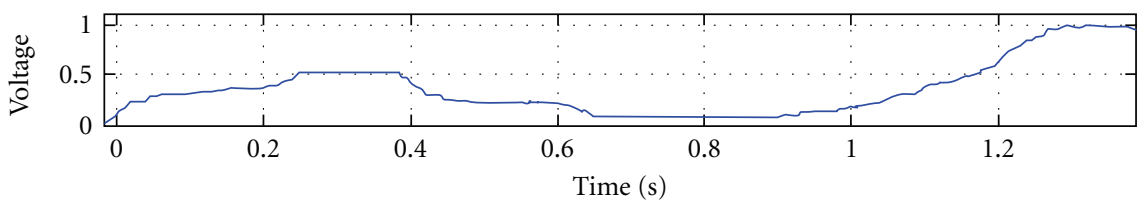

(e)

Figure 3: (a) Gait cycle aligned with normalized EMG voltages of (b) tibialis anterior, (c) gastrocnemius, (d) vastus lateralis, and (e) biceps femoris in a healthy case over a single gait cycle.

frequency-moments where the peak correlation between two signals occurs. Since this distribution returns a set of complex numbers, we assess the cross time-frequency analysis using the real part of the distribution. Our method uses the definition and properties of cross time-frequency analysis [22] to arrive at the quantitative metrics defined in Section 3.2. We define the joint time-moment and frequency-moment by use of the real part of cross time-frequency distribution, respectively,

$$
\begin{aligned}
& -t_{x_{1} x_{2}}(\omega)=\mathfrak{R}\left\{\frac{\int J_{x_{1} x_{2}}(t, \omega ; \phi) d t}{\int J_{x_{1} x_{2}}(t, \omega ; \phi) d t}\right\}, \\
& \omega_{x_{1} x_{2}}(t)=\mathfrak{R}\left\{\frac{\int \omega J_{x_{1} x_{2}}(t, \omega ; \phi) d \omega}{\int J_{x_{1} x_{2}}(t, \omega ; \phi) d \omega}\right\} .
\end{aligned}
$$

Because the cross time-frequency distribution is a complex representation, we find the normalized time and frequency moments by taking the real portion of the distribution. As we take the real part of the complex cross time-frequency distribution, the joint moment will collapse to instantaneous frequency and group delay in traditional auto time-frequency analysis if the signal pair $x_{1}(t)$ and $x_{2}(t)$ are identical. These joint moments in time and frequency domain are critically relevant for our study, where the time and frequency localized correlation is representative of muscular activation and force. For simplicity of mathematical expressions below, we assume that the cross timefrequency distribution $\left(J_{x_{1} x_{2}}(t, \omega)\right)$ is normalized to unity. Extending the definition of the joint time-and frequencymoments in (10), one can define following normalized joint 
time-and frequency-centers based on cross time-frequency distribution:

$$
\begin{aligned}
t_{x_{1} x_{2}} & =\Re\left\{\iint t \cdot J_{x_{1} x_{2}}(t, \omega) d \omega d t\right\}, \\
\omega_{x_{1} x_{2}} & =\Re\left\{\iint \omega \cdot J_{x_{1} x_{2}}(t, \omega) d \omega d t\right\} .
\end{aligned}
$$

The joint time center $\left(t_{x_{1} x_{2}}\right)$ and frequency center $\left(\omega_{x_{1} x_{2}}\right)$ enable us to identify the time and frequency centers of the pair of signals $x_{1}(t)$ and $x_{2}(t)$. In particular, the joint frequency center allows us to determine most significant overlapping frequency components between the signal pairs, a property utilized by one of our three metrics for evaluation. The applications of this definition and its experimental result are discussed in Section 4.1. Furthermore, the joint time and frequency center enable one to determine joint time duration $\left(T_{x_{1} x_{2}}\right)$ and frequency bandwidth $\left(\Omega_{x_{1} x_{2}}\right)$ as follows:

$$
\begin{aligned}
T_{x_{1} x_{2}}^{2} & =\Re\left\{\iint\left(t-t_{x_{1} x_{2}}\right)^{2} \cdot J_{x_{1} x_{2}}(t, \omega) d \omega d t\right\}, \\
\Omega_{x_{1} x_{2}}^{2} & =\Re\left\{\iint\left(\omega-\omega_{x_{1} x_{2}}\right)^{2} \cdot J_{x_{1} x_{2}}(t, \omega) d \omega d t\right\} .
\end{aligned}
$$

The traditional definitions of time duration and frequency bandwidth describe the degree of a distribution's spreading in the time-frequency domain. Likewise, the joint time-duration and joint frequency-bandwidth quantify the degree of spreading of the joint time-frequency signature in time and frequency domain respectively. In conjunction with the joint frequency center defined in (1), one can find significant differences between the healthy and unhealthy subjects' EMG signature in terms of the joint time duration $\left(T_{x_{1} x_{2}}\right)$ and joint frequency bandwidth $\left(\Omega_{x_{1} x_{2}}\right)$, which is discussed in Section 4.1.

3.2. Metric Selection. After the wave packet selection, it was desirable to obtain measures of overall gait health, as well as defining features, within the time-frequency analyses of the signals. By review and analysis, we have developed several parameters by which we gauge each subjects' overall fitness as it pertains to muscle activation for gait. These metrics are: the time-and frequency-bandwidth of the wave packet peak (to the $-3 \mathrm{~dB}$ point of the peak), the frequency center of the wave packet peak, and the percent of energy in the signal above $100 \mathrm{~Hz}$. Figure 4 shows the cross time-frequency distribution of a left and right EMG wave packet for single subject, with the two wave packets represented in both the time and frequency domain. The gait cycle is aligned in the figure, identifying the parallelism between muscular activation and the push-off phase of the gait cycle. The area above the white line denoted by (e) corresponds to the energy measured above $100 \mathrm{~Hz}$ through the course of a single contraction of the gastrocnemius. The focused area (f) contains the energy peak of the wave packet. This is the point at which the peak instantaneous energy in the wave packet occurs, as well as the time duration of the peak contraction and the frequency bandwidth of the same contraction.

It has been shown that higher mean frequency corresponds to a higher amount of force [23], and in the case of gait, this translates to cadence that is more succinct and deliberate, but can also indicate overexertion or inefficiency. The frequency center correlates to the maximal torque achieved within the muscle, specifically at the push-off instance in the gastrocnemius (see Figure 4). Therefore, it is desirable to examine this parameter as it relates to every footfall. This frequency center has a very distinct band around it, which corresponds to a much higher level of energy than otherwise present in the wave packet elsewhere. We measured and recorded this bandwidth, in both the time and frequency domains for each footfall as well as for the cross time-frequency analyses.

Through use of the time-frequency representation, we can easily determine the amount of energy within a specific range by merely integrating over the desired range. We then divide by the total energy contained in the signal to find a percentage.

$$
E_{x_{1} x_{2}}(\omega>2 \pi \cdot 100)=\frac{1}{E_{x}} \int_{\omega=2 \pi \cdot 100}^{\omega_{\max }} \int_{t=0}^{t_{\max }} J_{x_{1} x_{2}}(t, \omega) d t d \omega .
$$

This became our fourth metric; because more time spent when muscles are in this band correspond to faster contractions, and thus more powerful joint actuation. The four metrics are now defined, and in order to condense the large volume of metrics that exists upon time-frequency analysis of every footfall, a matrix must be defined to condense and intelligently present this information.

3.3. Definition of Matrix. As was previously described, Figure 2 shows the primary and secondary wave packet definitions. It was desirable to compare these wave packets to one another, as well as to perform analysis upon the primary wave packets themselves. To organize the comparison between the primary $\left(S_{1}, S_{2}\right)$ and secondary $\left(S_{1}^{\prime}, S_{2}^{\prime}\right)$ signals, a matrix was developed that can be used to represent the different parameters generated by each wave packet.

Equation (14) is the definition of our Spatiotemporal Discrepancy Matrix (SDM) proposed for this study. The cross time-frequency distribution metrics are in the upperright triangle, and the metric value contained in each slot is the value for that particular pair of wave packets' cross time-frequency analysis. The main diagonal contains the metrics for the autocorrelation of each prime wave packet, and the lower left triangle contains cross-correlation of the secondary wave packets. When performing analysis, this information is valuable for some cases of hypertensive subjects, as particularly in the case of the gastrocnemius, this muscle should not be active

$$
\tilde{S}=\left[\begin{array}{ccccc}
S_{11} & S_{21} & \cdots & S_{i-11} & S_{i 1} \\
S_{12}^{\prime} & S_{22} & \cdots & \ddots & S_{i 2} \\
\vdots & \vdots & \ddots & \vdots & \vdots \\
S_{1 j-1}^{\prime} & \ddots & \cdots & S_{i-1 j-1} & S_{i j-1} \\
S_{1 j}^{\prime} & S_{2 j}^{\prime} & \cdots & S_{i-1 j}^{\prime} & S_{i j}
\end{array}\right],
$$




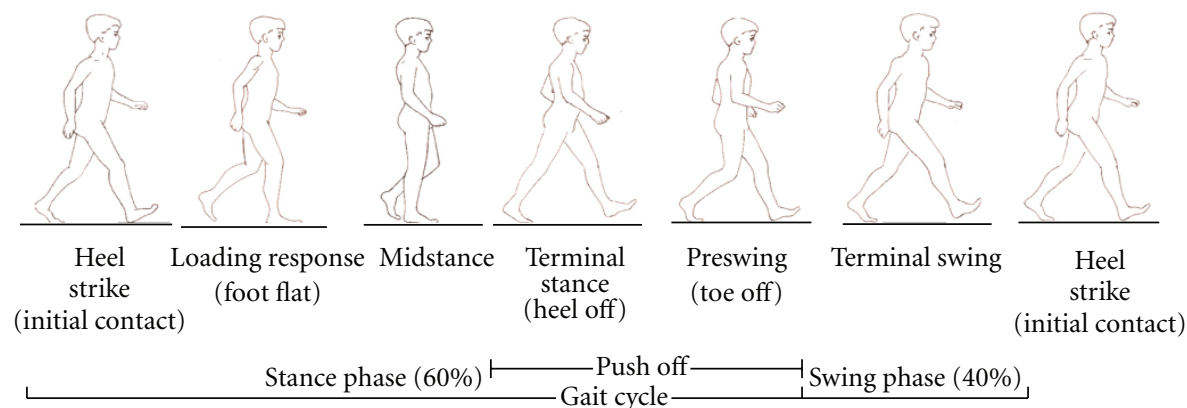

(a)

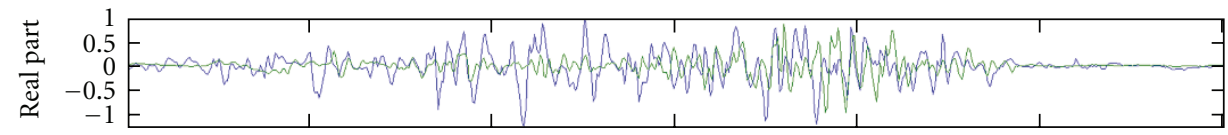

(b)

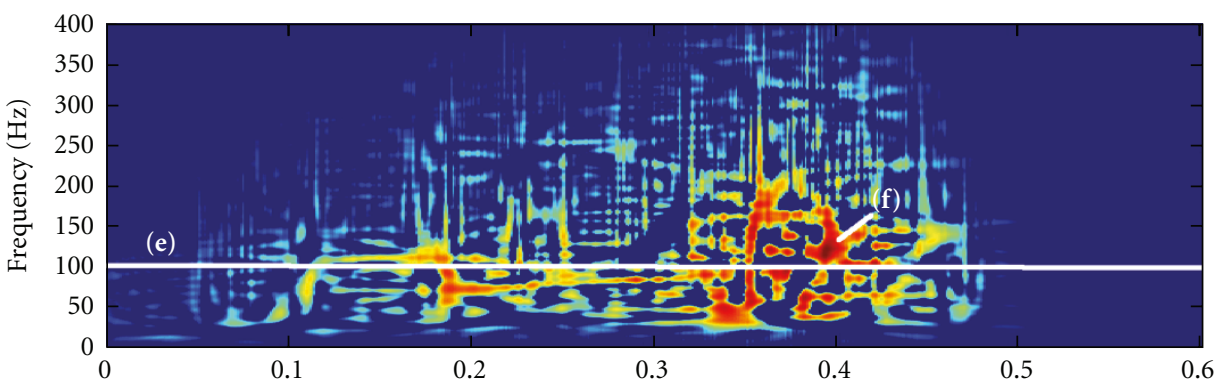

(c)

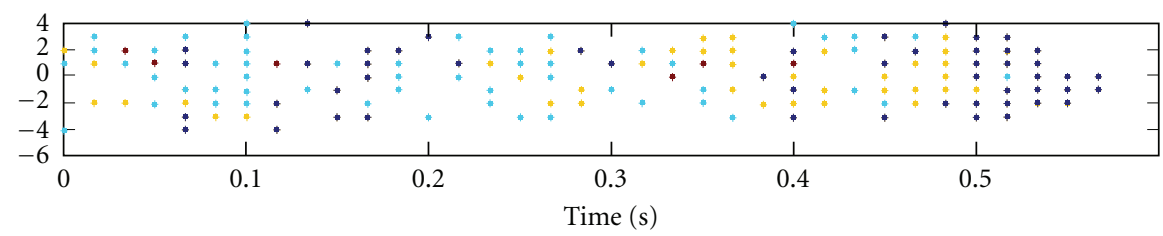

(d)

FIGURE 4: Time-aligned (a) gait cycle, (b) time-domain waveform of two EMG footfall wave packets, (c) cross time-frequency visualization of two EMG footfall wave packets, (d) line denoting separation of measure for energy above $100 \mathrm{~Hz}$, (e) location of peak energy and time-bandwidth measures, (f) recording of pressure data from time-synchronized mat.

where $S_{i j}, i=j$ is the time-frequency distribution, $S_{i j}, \quad i>j$ is the cross time-frequency distribution between $S_{i}$ and $S_{j}$, $S_{i j}^{\prime}, \quad i<j$ is the cross time-frequency distribution between $S_{i}^{\prime}$ and $S_{j}^{\prime}$, and $i=j=$ the total number of footfalls in the walk. Ergo, the values above the main diagonal correspond to the cross time-frequency analysis of the primary signals, and the values below the main diagonal correspond to the secondary signals. Each metric for each walk is organized into a single matrix, thus for a single walk, there are three matrices, as shown in Table 1.

These functions were defined in Section 3.1 by (12) and (13). The method by which we further condense the information and the use of the matrix is described in Section 4.2 .
TABLe 1: Example of matrices created due to single walk file assuming three footfalls in walk.

\begin{tabular}{llll}
\hline $\begin{array}{l}\text { Time } \\
\text { bandwidth } \\
(\mathrm{ms})\end{array}$ & $\begin{array}{l}\text { Frequency } \\
\text { bandwidth } \\
(\mathrm{Hz})\end{array}$ & $\begin{array}{l}\text { Frequency } \\
\text { center }(\mathrm{Hz})\end{array}$ & $\begin{array}{l}\text { Percent energy above } \\
100 \mathrm{~Hz}(\%)\end{array}$ \\
\hline$\widetilde{S_{i j}^{T}}=T_{S_{i} S_{j}}$ & $\widetilde{S_{i j}^{w}}=F_{S_{i} S_{j}}$ & $\widetilde{S_{i j}^{F}}=\omega_{S_{i} S_{j}}$ & $\begin{array}{l}\widetilde{S_{i j}^{E}}=E_{s_{i} s_{j}} \\
2 \pi \cdot 100)\end{array}$ \\
\hline
\end{tabular}

3.4. Application of Time-Frequency Analysis. Upon acquisition of data, we exported data for each walk to commaseparated value format and imported this information 
to a mathematical computation program. The walk data contained a matrix with time, $X, Y$, and pressure values for each sampled moment for which a pressure sensor was active. Using an algorithm that compares standard deviation within the $X$ and $Y$ coordinates to look for large gaps where footfalls occur, sections of stride were identified and their time instances flagged, as described in Section 2.2. These time instances correspond directly to the muscular activation during the gait cycle. After the selection of the signal using the footfall data, cross time-frequency information for each pair of footsteps (left, right, cross, all) was synthesized, and the metrics defined in Section 3.2 were stored in a matrix, defined in Section 3.3. This generated one matrix for each metric, for each walk, of which there are four per subject.

After creation of the matrices, data was aggregated and analyzed based on medical history. Subjects found to be without hypertension, diabetes, neuropathy, and a vitamin $\mathrm{D}$ deficiency were considered for the selection of our normal curve, as well as subjects under the age of 65 that had no gaitaltering conditions.

\section{Experimental Results}

4.1. Cross Time-Frequency Analysis of Gait EMG Wave Packets. To ascertain the significance of the findings of this paper, we will first examine two cases in detail, using the methodology described in previous sections. Figure 5 shows an example of one subject's gastrocnemius EMG signals in the time-domain during normal gait. The signals are separated as described in Section 2.2, beginning with a left footfall. These separated signals provide the data to which we will apply time-frequency and cross time-frequency analysis.

As was observed in Figure 2, for a healthy case the gastrocnemius muscle activates during the stance phase of its corresponding leg. To gain a more meaningful perspective on the data, we will use time-frequency analysis. Figure 6 shows a series of time-frequency visualizations of the signals denoted by $S_{1}-S_{6}$. The time-domain waveforms on which the time-frequency analysis is performed is the same across all six signals. The EMG signals were truncated as indicated in Figure 5.

It is clear from this set of representations that the gastrocnemius muscle activates for a large portion of the stance phase, culminating at push-off, around 350-400 ms. It is also worthwhile to notice the amount of content above $100 \mathrm{~Hz} . S_{3}$ is shown in more detail in Figure 7.

As discussed in Section 3.2, there are several metrics we identify and compare in each signal's time-frequency analysis. In this footfall, the frequency center lies at $f=$ $137 \mathrm{~Hz}(\mathrm{~d})$. This frequency center marks the frequency at which the highest instantaneous energy occurs within the wave packet. Fifty-one percent of the energy within the wave packet is above $100 \mathrm{~Hz}$, which signifies overall faster contraction throughout the wave packet. Also of note is the time duration, which is $48 \mathrm{~ms}$ for this wave packet. Shorter time durations correspond to a more succinct pushoff, and the frequency bandwidth corresponds to a more

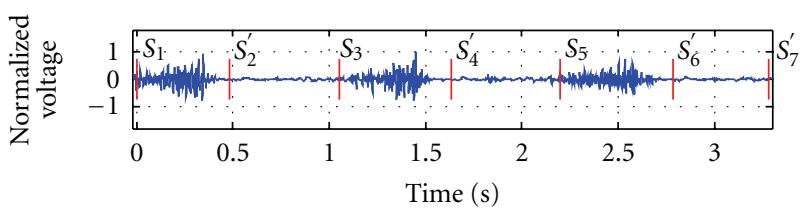

(a)

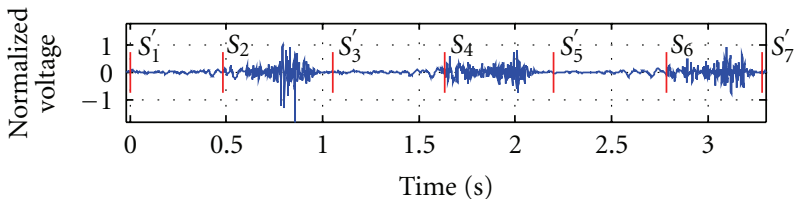

(b)

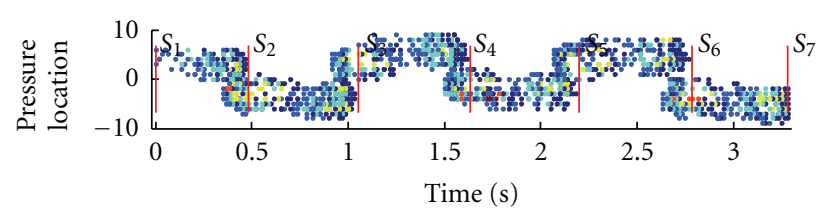

(c)

Figure 5: Signal representation of (a) left gastrocnemius (b) right gastrocnemius, and (c) pressure walkway recording, with swing phase divisions shown in red for nonhypertensive subject 001 walk 4 .

focused set of firings. Figure 8 shows the time-domain signal of the left and right gastrocnemius for another subject. Note the use of the muscle during the swing phase, during which the muscle should show very little use, if any at all. The swing phase for the muscle is marked by the secondary signals (the prime signals). There is also a large amount of muscle usage just before heel strike, where this rotation should be natural rather than orchestrated by the gastrocnemius.

The time-frequency distribution of wave packet 3 is provided in Figure 10. The peak energy frequency center is located at $f=68.2 \mathrm{~Hz}$, almost $50 \mathrm{~Hz}$ below the previous case. Also visible in the energy spectral density is a lower mean frequency. In addition, the amount of frequency content above $100 \mathrm{~Hz}$ is only $24.2 \%$, composing less than one fourth of the total energy. When comparing to Figure 7, the number of significant firings is also noteworthy. In the previous case, the energy up to the push-off point follows a smooth curve, while in this case firings occur rapidly, and are separated rather than contiguous. This becomes more visible in Figure 9.

In Figure 9, the discrepancies of the frequency center and energy above $100 \mathrm{~Hz}$ become apparent between the two cases. Note that in the second case, the maximum frequency of the peaks rarely exceeds $200 \mathrm{~Hz}$, and each wave packet shows short and pronounced time durations. In Figure 6, the peaks often exceed $200 \mathrm{~Hz}$, and are longer in duration in the lower-frequency bands.

After developing the metric definition for small cases, it is desirable to develop this information for a large number of cases. It is difficult to examine all cases visually, due to the number of individual time-frequency distributions 


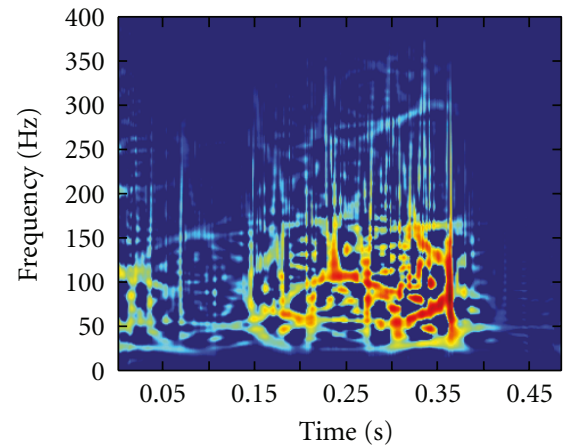

(a)

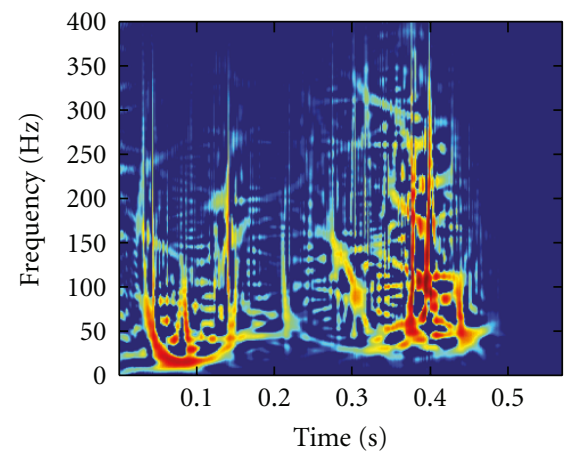

(d)

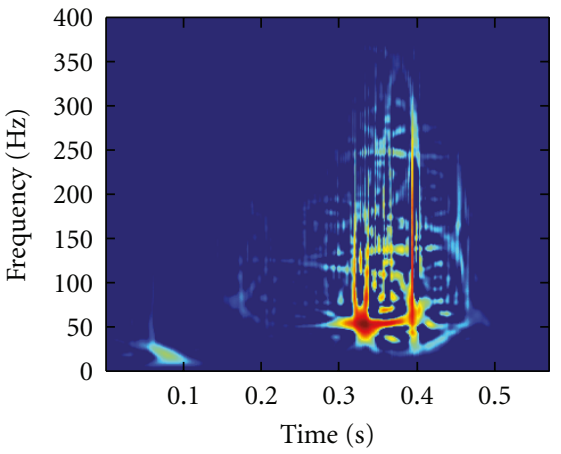

(b)

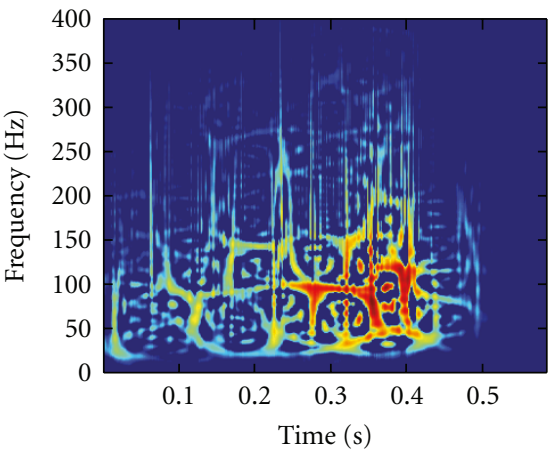

(e)

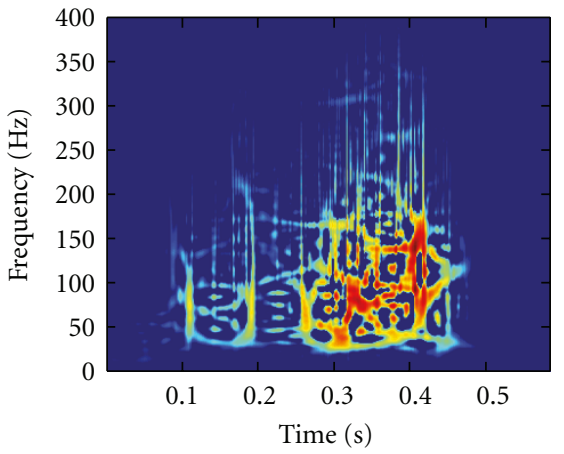

(c)

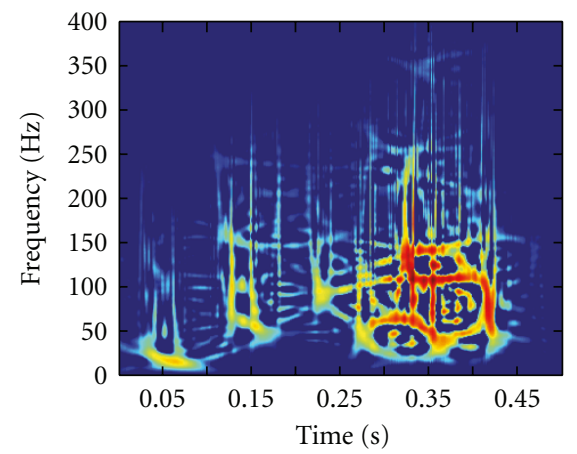

(f)

Figure 6: Individual muscle activation time-frequency visualizations of (a) $S_{1}$, (b) $S_{2}$, (c) $S_{3}$, (d) $S_{4}$, (e) $S_{5}$, and (f) $S_{6}$ from 001 walk 4 .

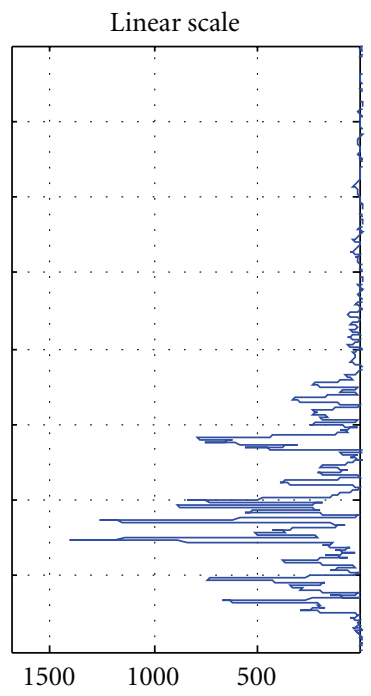

(a)

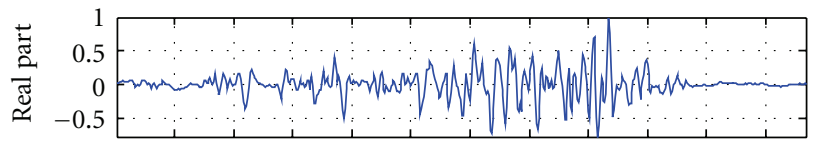

(b)

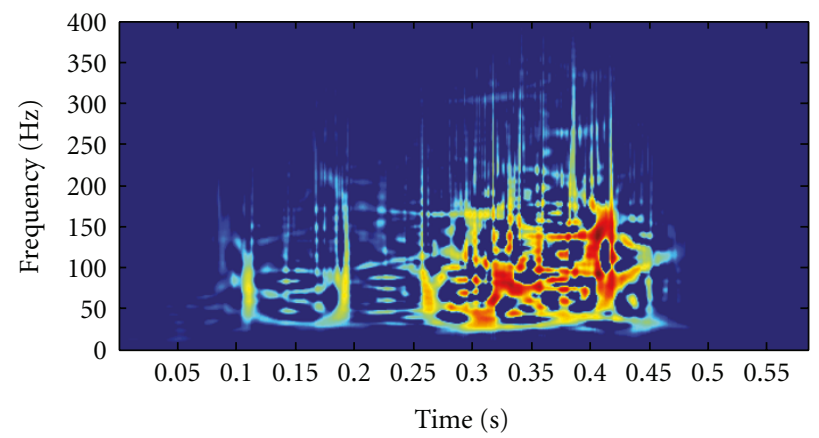

(c)

Figure 7: The (a) power spectral density, (b) time-domain waveform, (c) time-frequency visualization (d) energy peak of subject 001 walk $4 S_{3}$, over a single activation of the gastrocnemius.

performed on a per-footfall basis. The matrix described in Section 3.3 solves this problem by condensing the dense amount of information contained in a single time-frequency analysis into the primary metrics, which differ between subjects. By focusing only on the differentiating metrics, it is easy to develop a conclusion.
4.2. Spatiotemporal Discrepancy Matrix and Interpretation. The SDM developed contains all pertinent information for each metric as it pertains to each footfall. We compiled data for all subjects, whose medical report information was available at time of publication, therefore allowing us to compare averages across comorbidities. Equation (15) shows 


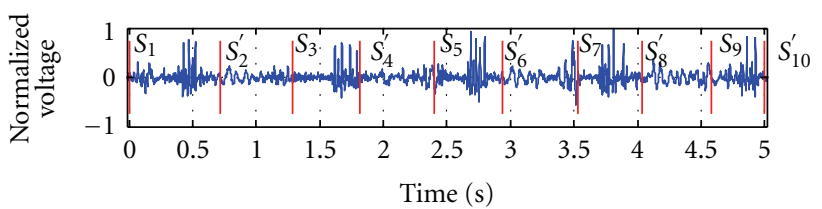

(a)

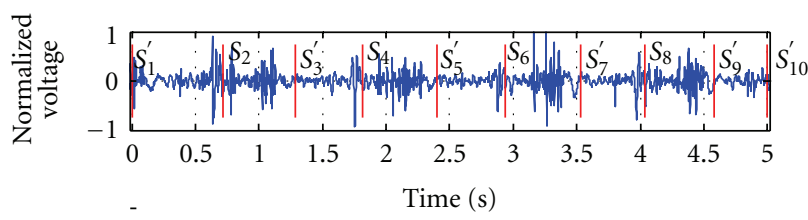

(b)

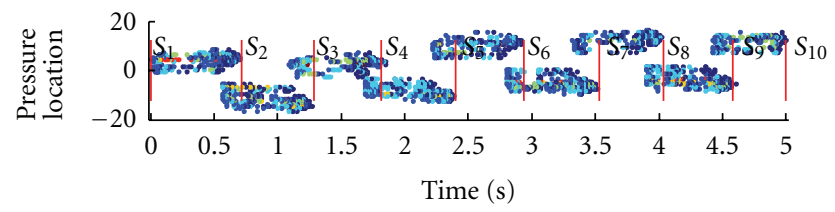

(c)

FIGURE 8: EMG signals of (a) left gastrocnemius (b) right gastrocnemius, and (c) pressure walkway recording, with swing phase divisions shown in red for hypertensive subject 004 walk 1. the matrix for the maximum frequency point for the case examined in Section 4.1

$\widetilde{S_{n h}^{F}}=\left[\begin{array}{rrrrrr}83.01 & 60.06 & 80.08 & 70.80 & 97.17 \\ 16.11 & 54.20 & 64.94 & 56.15 & 66.41 \\ 49.32 & 15.14 & 116.70 & 92.77 & 84.96 \\ 14.65 & 62.50 & 18.55 & 106.93 & 104.49 \\ 43.95 & 18.55 & 45.41 & 17.58 & 81.05\end{array}\right],(15)$

The main diagonal (boxed), as discussed in Section 3.3, contains the value of the frequency center for the autocorrelation wave packets shown in Figure 6. The upper-right triangle contains the values for the cross time-frequency analysis performed between two wave packets. Averaging these values together, we find an average frequency center of $81.32 \mathrm{~Hz}$ with a standard deviation of $\pm 19.40 \mathrm{~Hz}$. We then contrast this information with the second case we examined

$$
\widetilde{S_{h}^{F}}=\left[\begin{array}{cccccccccc}
\hline 43.46 & 48.34 & 52.25 & 45.90 & 38.09 & 74.71 & 77.64 & 24.90 \\
\cline { 5 - 8 } 11.72 & 44.43 & 78.18 & 73.73 & 60.06 & 83.98 & 100.10 & 62.01 \\
48.34 & 23.93 & 68.36 & 70.80 & 62.50 & 87.89 & 77.64 & 52.73 \\
8.79 & 33.20 & 19.53 & 78.61 & 66.41 & 75.20 & 66.89 & 100.59 \\
58.11 & 23.44 & 66.41 & 28.32 & 44.92 & 64.45 & 67.38 & 77.15 \\
21.48 & 37.60 & 24.90 & 31.25 & 24.90 & 88.38 & 79.10 & 89.36 \\
57.13 & 21.48 & 38.57 & 24.90 & 62.50 & 22.95 & 84.47 & 31.25 \\
11.72 & 35.64 & 21.48 & 45.41 & 27.83 & 31.25 & 19.53 & 21.97
\end{array}\right]
$$

It is immediately apparent that there are lower values for the frequency center on average. The mean value of the pertinent wave packets is $65.66 \mathrm{~Hz}$ with a standard deviation of $\pm 19.9 \mathrm{~Hz}$. When analyzing the matrices, it was determined that due to the nature of gait, one leg may perform slightly differently than the other. We therefore examined average metric values across all walks for the right foot, left foot, between feet, and as an average of all feet for the cross timefrequency distributions between each footfall, rather than a single lump sum average. Figure 11 shows these relations for 5 control subjects and 5 hypertensive subjects, with standard deviations marked.

As discussed in Section 4.1, the frequency centers, as well as the amount of energy above $100 \mathrm{~Hz}$ are measures used for assessing overall gait health. As is clear from the table, the nonhypertensive subjects have different values for the metrics in the intrafoot analyses. In addition, frequency center is lower for the control subjects, corresponding to less force in push-off activation. The percent of frequency content above $100 \mathrm{~Hz}$ is also significantly different, again corresponding to the overall force exacted by the muscle, and the amount of muscle use. When we look at the overall standard distribution of the metrics, we see in more detail the discrepancies between the groups.

4.3. Statistical Analysis of Control Subjects. Upon examination of the control subjects, normalized distribution characteristics were identified between the subjects, specifically those with no comorbidities, as seen from Figure 11. Therefore, we endeavored to create normal distributions based on the metrics of different groups' footfalls. To create a normal distribution for control subjects, we identified 10 subjects with no comorbidities or gait dysfunction. We used the methodology described in Section 3.4 to analyze both sets of faster-paced walks, and created a set of event metrics for each subject. For a subject with six footfalls in a single walk, there are six metrics generated for each of the left foot, right foot, and cross-foot measures. With two walks per subject, 


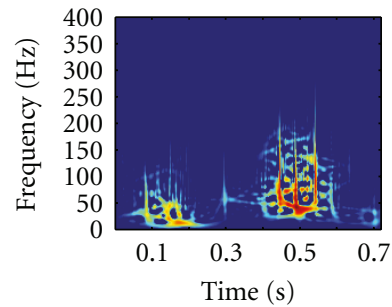

(a)

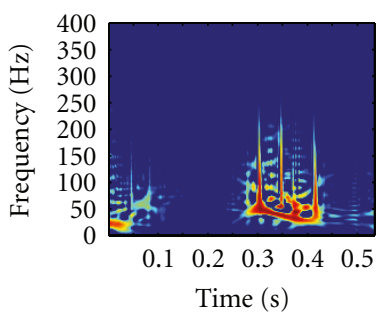

(e)

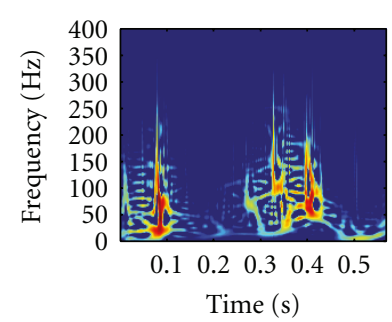

(b)

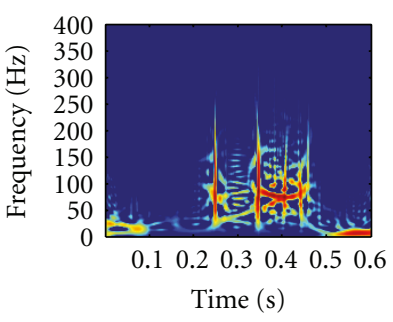

(f)

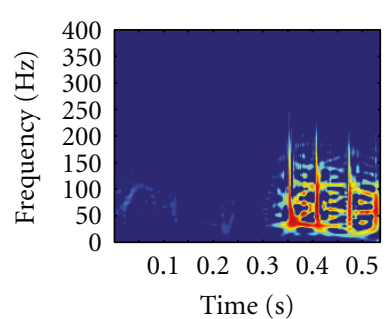

(c)

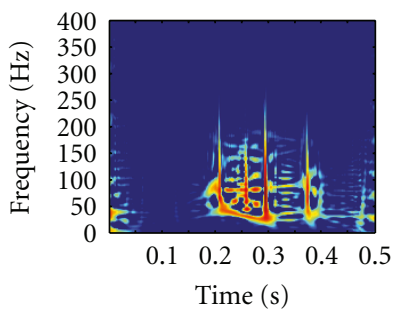

(g)

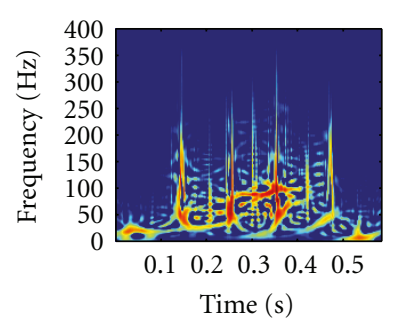

(d)

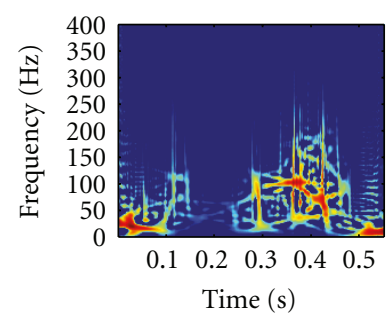

(h)

Figure 9: Previous subject's time-frequency visualizations for (a) $S_{1}$, (b) $S_{2}$, (c) $S_{3}$, (d) $S_{4}$, (e) $S_{5}$, (f) $S_{6}$, (g) $S_{7}$, and (h) $S_{8}$, highlighting overall similarity between distributions.

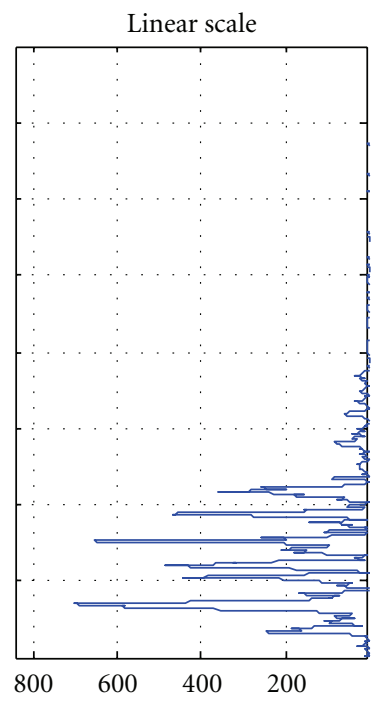

(a)

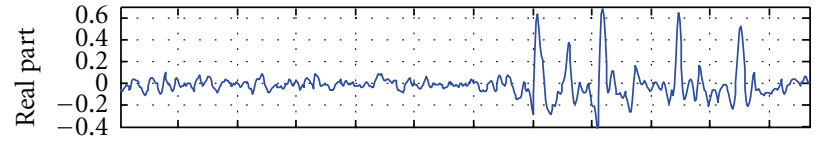

(b)

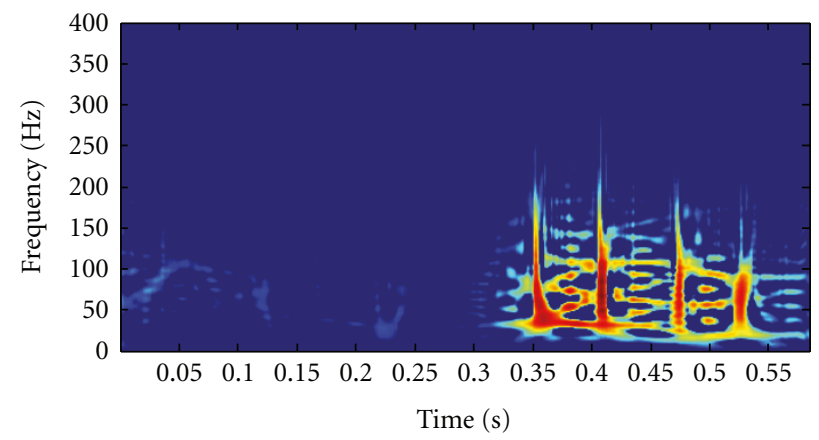

(c)

Figure 10: The (a) power spectral density, (b) time-domain waveform, (c) time-frequency visualization, and (d) energy peak for subject 003 walk four $S_{3}$.

when combined, there are 36 individual metric values for one subject. Our normal metric, therefore, is based on a large number of sampled data, two walks from each of ten subjects.

This normalized curve serves as a reference to which we can compare the recorded cases with significantly degenerated gait. Figure 12 shows the standard distribution of ten control cases, with no comorbidities, and several subjects whose metrics lie well outside the standard distribution. These graphs are organized across the four metrics, and are based on the left foot, right foot, cross-foot, and combinations (all) of each cross time-frequency pair given by each set of footfalls. Because the time-bandwidth (a) and frequencybandwidth (b) are both found in a similar manner, they are presented first, followed by the frequency center (c) and percent over $100 \mathrm{~Hz}$ (d). The size of population is relatively small in this pilot study, but we assumed normal distribution for a convenience of confidence level determination. With the calculated mean and standard deviation of the controlled 


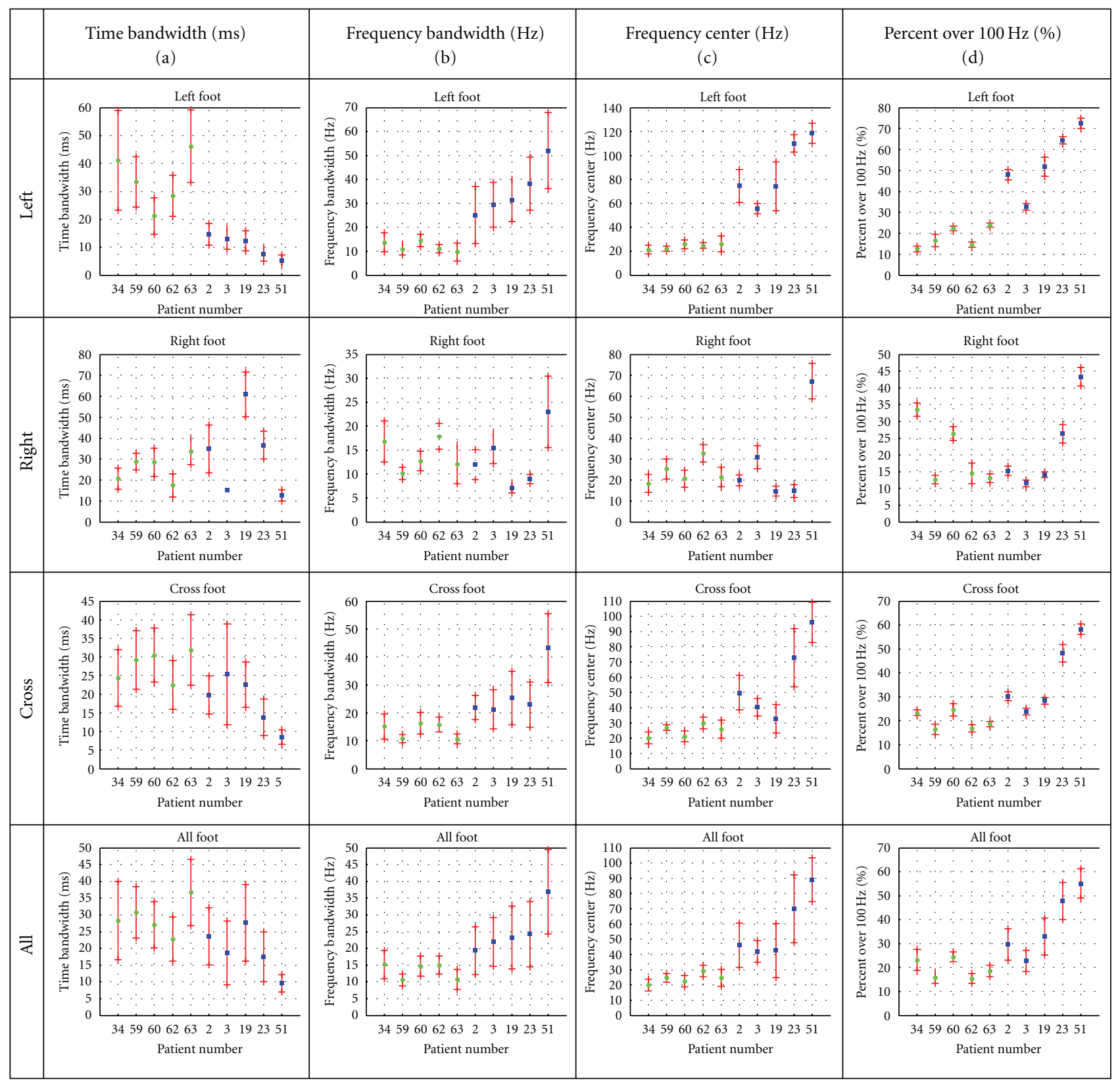

Figure 11: Means of cross-foot SDM for (a) time bandwidth, (b) frequency bandwidth, (c) frequency center, and (d) percent of energy above $100 \mathrm{~Hz}$; Mean values shown for control subjects are shown in green mean values for hypertensive cases are shown in blue standard deviations are shown on either side by red lines.

subjects displayed as a Gaussian standard distribution, the outlying subjects are organized such that they lie in the $x$-axis where their mean value occurs (for the respective metric). The $y$ values of the outlying subjects are arbitrary, and are organized to allow for legibility of the corresponding subject numbers. Mean and variance of individual distribution are provided in each sub-figure in Figure 12.

Upon investigation of the outlying subjects' medical histories, we find a large number of comorbidities present in varying degrees in each of the cases. Several of the medical conditions of the outlying subjects are listed in notified by the other measures. It is a notable fact that the frequencyrelated parameters in Figure 12 are obtained by cross timefrequency analysis that captures time-localized cross spectral characteristics of signals, which is proposed in this paper Table 2 .

The majority of subjects have hypertension, and many exhibit other comorbidities that exacerbate the symptoms and complications of hypertension. The standard deviations of distribution are marked in Figure 12, to show the confidence with which the normal distribution identifies outlying subjects. One can find that both the time bandwidth and 


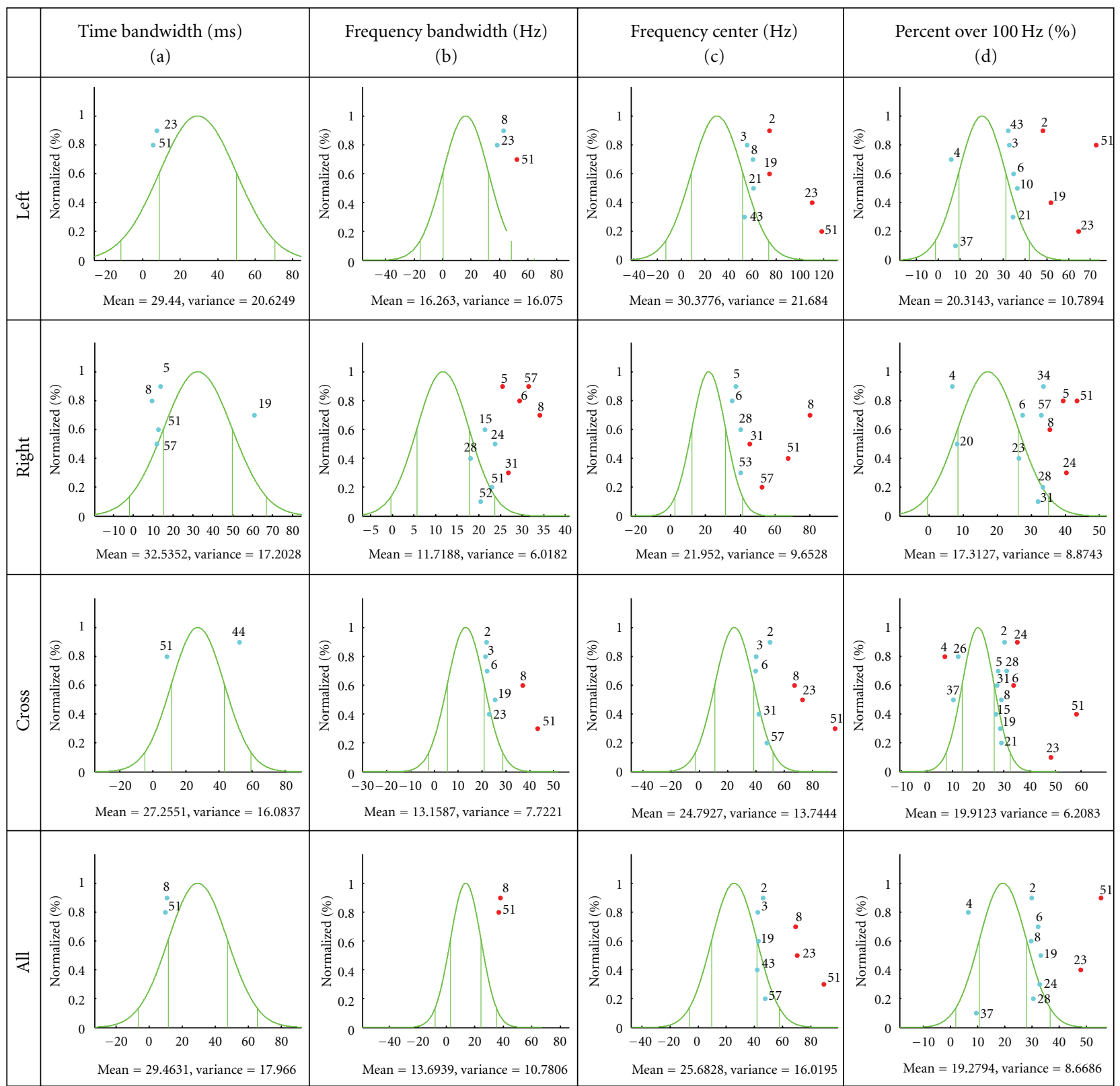

Figure 12: Cross-foot Gaussian distribution functions based on 10 control cases for (a) time bandwidth, (b) frequency bandwidth, (c) frequency center, and (d) percent energy above $100 \mathrm{~Hz}$ with outlying subjects marked in blue ( $1 \sigma$ outside) and red ( $2 \sigma$ outside).

frequency bandwidth are less effective in their assessment of outlying subjects, while the frequency center and percent above $100 \mathrm{~Hz}$ measures have much lower variance in the control distribution. However, the cas-es identified by both the time and frequency bandwidths are also identified by the other measures. It is a notable fact that the frequencyrelated parameters in Figure 12 are obtained by cross timefrequency analysis that captures time-localized cross spectral characteristics of signals, which is proposed in this paper.

As is apparent in the table, several of the outlying subjects had multiple comorbidities that jointly affect gait. This fact corroborates the hypothesis that specific, measurable quantities can be used to identify certain comorbidities that affect gait. Identifiable measures of change include a higher frequency center for hypertensive subjects, more energy over $100 \mathrm{~Hz}$, larger-frequency bandwidth, and a smaller time-bandwidth. These findings corroborate the existing knowledge base, but the trends in this case are entirely quantifiable due to the use of the cross time-frequency analysis technique. It should be noted, however, that not all the hypertensive cases were identified at this time using the metric, but those cases that were not identified typically had fewer comorbidities than those identified. This result can be interpreted such that the proposed methodology is suited towards identification of severe cases of hypertension or other comorbidities, rather than strict identification of 
TABLE 2: List of comorbidities for subjects that lie outside the normal distribution of metrics.

\begin{tabular}{|c|c|c|c|c|c|c|c|c|c|}
\hline $\begin{array}{l}\text { Subject } \\
\text { number }\end{array}$ & Age & Hyper-thyroid & Hyper-tension & Osteoporosis & Arthritis & Neuropathy & Diabetes & Vitamin-D deficient & $\begin{array}{l}\text { Coronary } \\
\text { artery disease }\end{array}$ \\
\hline 2 & 78 & Yes & Yes & & Yes & Yes & & Yes & \\
\hline 4 & 70 & Yes & Yes & & Yes & & Yes & & \\
\hline 5 & 81 & Yes & Yes & Yes & Yes & & & & \\
\hline 6 & 87 & & Yes & Yes & & & & & \\
\hline 8 & 80 & & Yes & & & & & & \\
\hline 15 & 87 & & Yes & Yes & Yes & & & & \\
\hline 19 & 86 & & & & Yes & Yes & & & Yes \\
\hline 21 & 78 & & Yes & & & & & & \\
\hline 23 & 93 & & Yes & & Yes & & & & \\
\hline 24 & 87 & Yes & Yes & Yes & Yes & & & & \\
\hline 26 & 77 & & Yes & & & Yes & Yes & & Yes \\
\hline 28 & 75 & & Yes & & & & & & \\
\hline 31 & 86 & & Yes & Yes & & Yes & & & \\
\hline 37 & 85 & & Yes & & & & & & Yes \\
\hline 51 & 81 & & Yes & & & Yes & & Yes & \\
\hline
\end{tabular}

hypertension. It is necessary for us to investigate statistical correlation between hypertension and gait problems in future. Also, due to the accuracy of the medical records provided, and a general difficulty in assessing hypertension, it is unknown which subjects' hypertension was controlled, uncontrolled, or otherwise more extreme as compared to other cases. And although the proposed use of cross timefrequency analysis may be helpful for the identification of these comorbidities, it is still necessary for a trained clinician to assist in the diagnosis and assessment of health in clinical applications. Ultimately, the goal of this methodology is to assist physicians in the identification of comorbidities, and then have their presence corroborated by clinical diagnosis.

\section{Conclusion}

Because certain comorbidities, directly affect gait, analysis of the electromyographic signals of the muscles responsible for gait is useful for identifying signs of these comorbidities, as well as deficiencies in gait patterns. Doctors using electromyography, however, are subject to error, and most EMG signals have very dense information packing, making it difficult to ascertain certain details. This research has proposed a solution using time-frequency analysis of gaitrelated EMG information that uses four quantitative metrics to distinguish hypertensive and nonhypertensive cases.

Through careful selection of each wave packet, we have created an algorithm that can accurately compare parameters of wave packets on an individual subject basis. This method relies on the synchronization of footfall data with collected electromyographic data. This synchronization makes possible the cadence separation to allow for crosssubject comparison of muscle activations. After separation, cross time-frequency analyses are performed using a reduced interference kernel on several cases to determine specific markers that identified discrepancies between hypertensive and nonhypertensive subjects. These metrics, obtainable through the time-frequency analysis of footfall wave packets, are the time duration of $25 \%$ of the peak of the signal, the frequency at which the peak instantaneous energy occurs, and the ratio of energy above $100 \mathrm{~Hz}$ to the energy of the wave packet. Using the cross time-frequency analysis, we can uniquely compare sets of muscle activation wave packets and determine the weighted value of the interaction between muscles.

In this paper, we analyzed two cases in detail, substantiating the basis for our metric selection. We then developed our matrix to compare these individual metrics, and using this matrix, we compared different subjects to one another. The resulting differences show that upon analysis and comparison between control cases and hypertensive cases, all four metrics identify discrepancies within one standard deviation. This quantitative analysis of gait degeneration is unique in its independence from intuitive assessment, as well as the accuracy of muscle activation selection ensured by synchronization of gait with EMG acquisition. As work continues on this ongoing project, we hope to substantiate our claims by processing and analyzing a greater number of subjects. We desire to analyze at least 80 subjects, increasing the efficacy of our results. This paper examined a very specific set of gastrocnemius activations during only the stance phase, but recordings exist for three additional muscles, as well as the opportunity to analyze and categorize abnormalities in the swing phase for the gastrocnemius muscle, as well as the other muscles. In this preliminary study, analysis focused on the gastrocnemius muscle during the stance phase, but not during the swing phase, leaving a large portion of information untapped. As the size of our database increases, we will continue to develop and identify new methods and metrics by which to identify those subjects with comorbidities, and while these results are 
promising, more substantiation is necessary before we begin investigating reliable clinical application.

\section{Acknowledgments}

This paper is sponsored by Palmetto Health and SeniorSMART center. The authors appreciate the cooperation of the staff for experiments and data collection in the Department of Geriatrics, Palmetto Health, as well as Still Hopes Retirement Community. The authors would also like to thank Mr. Daniel Joseph Hood for his artistic work in this paper.

\section{References}

[1] P. Kannus, J. Parkkari, S. Koskinen et al., "Fall-induced injuries and deaths among older adults," Journal of the American Medical Association, vol. 281, no. 20, pp. 1895-1899, 1999.

[2] http://www.cdc.gov/injury/wisqars/index.html, January 2010.

[3] M. C. Hornbrook, V. J. Stevens, D. J. Wingfield, J. F. Hollis, M. R. Greenlick, and M. G. Ory, "Preventing falls among community-dwelling older persons: results from a randomized trial," Gerontologist, vol. 34, no. 1, pp. 16-23, 1994.

[4] A. Bergland and T. B. Wyller, "Risk factors for serious fall related injury in elderly women living at home," Injury Prevention, vol. 10, no. 5, pp. 308-313, 2004.

[5] A. J. Campbell, J. Reinken, B. C. Allan, and G. S. Martinez, "Falls in old age: a study of frequency and related clinical factors," Age and Ageing, vol. 10, no. 4, pp. 264-270, 1981.

[6] M. E. Tinetti, M. Speechley, and S. F. Ginter, "Risk factors for falls among elderly persons living in the community," The New England Journal of Medicine, vol. 319, no. 26, pp. 1701-1707, 1988.

[7] F. Englander, T. J. Hodson, and R. A. Terregrossa, "Economic dimensions of slip and fall injuries," Journal of Forensic Sciences, vol. 41, no. 5, pp. 733-746, 1996.

[8] M. E. Tinetti and C. S. Williams, "The effect of falls and fall injuries on functioning in community-dwelling older persons," Journals of Gerontology, vol. 53, no. 2, pp. M112M119, 1998.

[9] J. A. Stevens, P. S. Corso, E. A. Finkelstein, and T. R. Miller, "The costs of fatal and non-fatal falls among older adults," Injury Prevention, vol. 12, no. 5, pp. 290-295, 2006.

[10] J. F. Bean, D. K. Kiely, S. Herman et al., "The relationship between leg power and physical performance in mobilitylimited older people," Journal of the American Geriatrics Society, vol. 50, no. 3, pp. 461-467, 2002.

[11] D. A. Skelton, C. A. Greig, J. M. Davies, and A. Young, "Strength, power and related functional ability of healthy people aged 65-89 years," Age and Ageing, vol. 23, no. 5, pp. 371-377, 1994.

[12] J. M. Hausdorff, T. Herman, R. Baltadjieva, T. Gurevich, and N. Giladi, "Balance and gait in older adults with systemic hypertension," American Journal of Cardiology, vol. 91, no. 5, pp. 643-645, 2003.

[13] W. J. Williams, "Reduced interference distributions: biological applications and interpretations," Proceedings of the IEEE, vol. 84, no. 9, pp. 1264-1280, 1996.

[14] P. Bonato, S. H. Roy, M. Knaflitz, and C. J. De Luca, “Time frequency parameters of the surface myoelectric signal for assessing muscle fatigue during cyclic dynamic contractions," IEEE Transactions on Biomedical Engineering, vol. 48, no. 7, pp. 745-753, 2001.
[15] G. De Michele, S. Sello, M. C. Carboncini, B. Rossi, and S.-K. Strambi, "Cross-correlation time-frequency analysis for multiple EMG signals in Parkinson's disease: a wavelet approach," Medical Engineering and Physics, vol. 25, no. 5, pp. 361-369, 2003.

[16] Y.-J. Shin, D. Gobert, S.-H. Sung, E. J. Powers, and J. B. Park, "Application of cross time-frequency analysis to postural sway behavior: the effects of aging and visual systems," IEEE Transactions on Biomedical Engineering, vol. 52, no. 5, pp. 859868, 2005.

[17] R. F. M. Kleissen, M. C. A. Litjens, C. T. M. Baten, J. Harlaar, A. L. Hof, and G. Zilvold, "Consistency of surface EMG patterns obtained during gait from three laboratories using standardised measurement technique," Gait and Posture, vol. 6, no. 3, pp. 200-209, 1997.

[18] M. Folstein, S. Folstein, and P. McHugh, Mini Mental State Examination, Psychological Assessment Resources (PAR), 1975.

[19] L. Cohen, Time-Frequency Analaysis, Prentice-Hall, Upper Saddle River, NJ, USA, 1st edition, 1995.

[20] C. Zheng, S. E. Widmalm, and W. J. Williams, "New timefrequency analyses of EMG and TMJ sound signals," in Images of the Twenty-First Century. Proceedings of the 11th Annual International Conference of the IEEE Engineering in Medicine and Biology Society, Engineering in Medicine and Biology Society, pp. 741-742, November 1989.

[21] J. Jeong and W. J. Williams, "Kernel design for reduced interference distributions," IEEE Transactions on Signal Processing, vol. 40, no. 2, pp. 402-412, 1992.

[22] Y. J. Shin, E. J. Powers, and W. M. Grady, "On definition of cross time-frequency distribution function," in Advanced Signal Processing Algorithims, Architectures, and Implementations, Proceedings of SPIE, pp. 9-16, San Diego, Calif, USA, July 2000.

[23] S. Karlsson and B. Gerdle, "Mean frequency and signal amplitude of the surface EMG of the quadriceps muscles increase with increasing torque-a study using the continuous wavelet transform," Journal of Electromyography and Kinesiology, vol. 11, no. 2, pp. 131-140, 2001. 